\title{
Escore de lesões e transtornos de locomoção de vacas Holandesas em instalações free-stall com diferentes tipos de cama
}

\author{
Injury scores and locomotor disorders of Holstein cows in a free-stall facility with different beds
}

Daiane Cecchin - Alessandro Torres Campos • Maria de Fátima Ávila Pires •

Francine Aparecida Sousa - Pedro Ivo Sodré Amaral - Tadayuki Yanagi Junior •

Suane Alves Ferreira - Myriam Cristiane Morais Souza - Diego Cecchin

D Cecchin (Autor para correspondência) - AT Campos - FA

Sousa - PIS Amaral - T Yanagi Júnior

Universidade Federal de Lavras (UFLA), Lavras, MG,

Brasil.

email: daianececchin@yahoo.com.br

\section{MFA Pires}

Empresa Brasileira de Pesquisa Agropecuária, Embrapa Gado de leite.

\section{SA Ferreira - MCM Souza}

Universidade Presidente Antônio Carlos (UNIPAC), Juiz de Fora, MG, Brasil.

\section{Cecchin}

Universidade de Passo Fundo (UPF), Passo Fundo, RS, Brasil.

Recebido: 09 de Outubro, 2015 - Revisado: 13 de Dezembro, 2015 - Aceito: 13 de Dezembro

Resumo Objetivou-se avaliar lesões de jarrete e joelho e transtornos de locomoção em 36 vacas Holandesas multíparas confinadas em sistema modelo free-stall com dois tipos de camas, na Embrapa Gado de Leite do município de Coronel Pacheco/MG. Empregaram-se camas de borracha composta e camas de areia. As lesões de jarrete e joelho e transtornos de locomoção foram avaliados em escores de gravidade. Observou-se que não houve diferença entre os escores observados no início e ao final do período experimental para as lesões de joelho em ambos os materiais de cama, o mesmo foi observado para lesões de jarrete, em animais alojados sobre cama de areia. As lesões se mantiveram com os mesmos escores durante o período estudado, demonstrando assim que as duas camas oferecem a mesma condição de conforto em relação a lesões e saúde do casco, pelo menos, em curto prazo de tempo.

Palavras-chave: bem-estar, confinamento, construções rurais, jarrete

\section{Introdução}

O tempo em que os bovinos que permanecem deitados é de extrema importância porque representa os momentos de descanso e a diminuição deste tempo pode resultar em alterações fisiológicas, afetando a saúde e o desempenho destes animais. Vacas leiteiras passam aproximadamente a metade de suas vidas deitadas, com média de 12 a 14 horas $\mathrm{dia}^{-1}$ (Boone et al 2009). O comportamento de descanso é ainda mais relevante em

\begin{abstract}
The aim of the present study was to evaluate hock and knee injuries and locomotor disorders in 36 multiparous Holstein cows confined in a free-stall model system with two types of beds at Embrapa Dairy Cattle in the city of Coronel Pacheco / MG. Rubber composite beds and sand beds were compared and the hock and knee injuries and locomotor disorders were assessed for severity scores. There was no difference between the scores or hock lesions observed at the beginning and end of the trial period for knee injuries in both beds. The two beds offer the same conditions of comfort, injury rates, and hull health, at least in the short term.
\end{abstract}

Keywords: welfare, confinement, rural buildings, hock

sistemas em que há restrição à movimentação dos animais, como nos confinamentos.

O confinamento surgiu como uma alternativa para o aumento da produtividade dos bovinos, propiciando novas oportunidades para manejar rebanhos leiteiros, dando origem, entretanto, a novos desafios a serem vencidos (Perissinotto et al 2009). O material utilizado como cama é considerado um desses desafios, podendo causar problemas para saúde e bem estar dos animais quando o mesmo não é adequado. 
Segundo Mitev et al (2012), a cama deve ser confortável o suficiente para garantir um descanso adequado e, consequentemente, melhorar o conforto e bem-estar dos animais. De acordo com Castro et al (2013), o uso de novos materiais pode ser encarado como uma tentativa de melhorar o ambiente no interior das instalações, porém a relação entre o ambiente e bem-estar dos animais deve ser cuidadosamente avaliada para que não ocorram perdas produtivas relacionadas a utilização de materiais inadequados .

As características individuais do animal, o manejo, o dimensionamento e o material a ser utilizado como cama em instalações tipo free-stall, podem influenciar o comportamento de descanso, o tempo de permanência e o índice de ocupação das baias (Carreira et al 2009). Pode-se esperar como consequência, alterações nas frequências de lesões nos cascos, jarretes e joelhos dos animais, bem como a ocorrência de mastite (Boone et al 2009).

Baias com dimensionamento e materiais adequados propiciam a minimização do estresse, bem como a redução dos níveis de claudicação, além de permitir que os animais expressem uma variedade de comportamentos, incluindo o de locomoção, com implicações positivas no ambiente social. No entanto, um estábulo mal dimensionado, além de causar lesões, como relatado por Cerqueira et al (2011), agrava a competição por recursos (alimento, água e áreas de repouso), sendo um fator de perturbação, gerando comportamentos agressivos e instabilidade social no rebanho.

A lesão de jarrete é um termo que engloba desde a ocorrência de pelos danificados ou perda dos mesmos, até pele ferida ou irritada, crostas e edemas (Rutherford et al., 2008). Solução de continuidade da pele e aumento de volume no local do jarrete são considerados lesões mais graves do que áreas com perda de pelo somente (Barberg et al 2007; Lombard et al 2010).

Lesões no joelho e jarrete são comuns em animais criados em galpões free-stall, especialmente aqueles que usam colchões (Lombard et al 2010). Fulwider et al (2007), estudando 100 fazendas, relataram que mais de $80 \%$ das vacas alojadas em baias com cama de colchão tinham lesões nos jarretes. Os autores observaram que as vacas alojadas em baias com cama de areia tinham pouca ou nenhuma lesão de jarrete.

O escore de locomoção foi positivamente correlacionado com lesões de jarrete (Fulwider et al 2007; Kielland et al 2009; Barker et al 2010). As lesões de jarrete podem levar a distúrbios da marcha, devido à restrição mecânica de flexão da articulação, infecção local ou dor associada à lesão (Potterton et al 2011). Vacas com dificuldade de locomoção deitam-se durante longos períodos (Chapinal et al 2010), aumentando assim o tempo de contato com a superfície e, por consequência, aumentando o potencial para desenvolvimento ou agravamento de lesões
(Potterton et al 2011). Segundo Weary et al (2009), alterações no comportamento podem indicar que o animal está sob estresse ou ocorrencia de alguma doença.

O período da vida util, no free- stall, dos tapetes ou colchões também está relacionado com o desenvolvimento de lesões, pois, materiais mais velhos mostraram-se menos abrasivos, diminuindo o desenvolvimento de lesões (Potterton et al 2011).

Sendo assim, o objetivo deste trabalho foi avaliar a efeito de dois diferentes tipos de cama, borracha e de areia sobre os escores de lesões de joelho, jarrete e locomoção de vacas da raça holandesa mantidas em sistema de confinamento modelo free-stall.

\section{Material e Métodos}

O trabalho foi desenvolvido nas instalações do Sistema Intensivo de Produção de Leite (SIPL) da Embrapa Gado de Leite, situado no Município de Coronel Pacheco, Zona da Mata de Minas Gerais, localizado à latitude de $21^{\circ} 33^{\prime} 22^{\prime \prime}$ Sul e à longitude de $43^{\circ} 06^{\prime} 15^{\prime \prime}$ 'Oeste, com altitude de $414 \mathrm{~m}$. O clima da região, segundo a classificação de Köppen, é Cwa, ou seja, clima quente, temperado chuvoso, com estação seca no inverno e com verão quente e chuvoso.

$\mathrm{O}$ experimento foi realizado em um galpão de confinamento modelo free-stall, com $37,1 \mathrm{~m}$ de comprimento, 36,0 m de largura, 7,0 m de altura no centro, e 3,5 m de pé-direito, com capacidade para alojar 80 animais adultos divididos em quatro lotes. Neste trabalho foram utilizados dois lotes.

O galpão apresenta as seguintes características: abertura total nos quatro lados, pilares de concreto prémoldado, piso de concreto frisado para melhor escoamento da água e dejetos, corredor central de alimentação medindo 4,5 m de largura, e corredor de manejo para condução dos grupos para a sala de ordenha de $4,1 \mathrm{~m}$.

A cobertura é feita com telhas de fibrocimento, sendo a região da cumeeira descoberta, visando obter ventilação por efeito termossifão. Os oitões são fechados com telhas de fibrocimento, instaladas na posição vertical, com o objetivo de reduzir a incidência de radiação solar direta no interior da instalação.

Foram empregadas, duas alas do free-stall, contendo 18 camas individuais (stalls), recobertas com borracha constituída por uma capa de poliéster recheada com roletes entremeados de borracha picada (subproduto da vulcanização de pneus) e outra ala composta por 18 camas recobertas com areia. As ordenhas eram realizadas três vezes ao dia, às $5 \mathrm{~h}$ $30 \mathrm{~min} ., 13 \mathrm{~h}$ e às $21 \mathrm{~h}$ (em sala de ordenha modelo espinha de peixe, contígua ao free-stall).

Foram observadas 36 vacas multíparas, de raça holandesa puras de origem, com produtividade média de $29,2 \pm 7,77 \mathrm{~kg} \mathrm{dia}^{-1}$, com massa corporal média de $560 \pm 64,1$ 
kg. A alimentação volumosa era constituída de silagem de milho, e $2 \mathrm{~kg}$ de capim tifton (Cynodon nlemfuensis) picado por animal, com o intuito de aumentar o teor de fibras da dieta, visando reduzir a incidência de acidose nos animais. $\mathrm{O}$ concentrado fornecido (13 kg por animal $\mathrm{dia}^{-1}$ ) continha $22 \%$ de proteína bruta (PB) e era acrescido de $100 \mathrm{~g}$ de bicarbonato de sódio. A água era disponível ad libitum. A alimentação era fornecida nos seguintes horários: 7, 10, 14, 16 e $21 \mathrm{~h}$.

Os animais foram mantidos na instalação por um período de 40 dias, durante os meses de abril e maio de 2012.

As lesões nos joelhos e jarretes foram pontuadas segundo o escore estabelecido por Norring et al. (2008). A severidade das lesões foi registrada em uma escala de seis pontos (zero: sem lesão; um: perda ou quebra de pelos; dois: pele nua; três: calos; quatro: vermelhidão da pele; cinco: cortes abertos). Os dados de lesões de jarretes e joelhos foram coletados por meio de aferição visual, no início do experimento (com oito dias de alojamento) e término (30 dias após a primeira avaliação).

A avaliação dos cascos foi realizada no início e ao final do experimento observando-se o deslocamento dos animais e utilizando-se um escore de locomoção de 5 pontos, estabelecido por Sprecher et al. (1997): escore 1: vaca normal - movimentação normal; escore 2: claudicação leve- postura arqueada ao andar, porem a marcha permanece normal; escore 3: claudicação moderada - postura arqueada tanto em estação quanto andando, marcha irregular com um ou mais membros afetados; escore 4: claudicação - postura arqueada e marcha com um passo de cada vez, favorecendo um ou mais membros; escore 5: claudicação severa incapacidade ou relutância extrema para suportar o peso de um ou mais membros.

Para a análise estátistica dos dados foi utilizado o teste de Mann-Whitney, assim como descrito por Norring et al (2008), para atestar diferenças entre os materiais quanto à gravidade das lesões dos jarretes, joelhos e locomoção usando a diferença entre a pontuação final e inicial do experimento.

\section{Resultados e Discussão}

Não houve diferença estatística $(\mathrm{P}<0,05)$ entre os escores observados no início e ao final do período experimental tanto para as lesões de joelho, quanto de jarrete em ambos os materiais de cama (Tabela 1).

Os resultados demonstram que não houve diferença entre os materiais, sendo assim, o colchão de borracha promoveu a mesma condição de conforto (em relação às incidências de lesões) para os animais que estavam alojados em baias com cama de areia. Durante esse intervalo de tempo em que os animais estiveram expostos a esses materiais como recobrimento de cama, os resultados encontrados no presente estudo se opõem aos obtidos por Espejo et al (2006), Fulwider et al (2007) e Norring et al (2008) que demostram diferença entre os tipos de cama (borracha e areia). Os autores relataram menor incidência de lesões nos jarretes quando utilizou-se areia como material de cama, possivelmente pelo fato da areia moldar-se à forma do animal, reduzindo o atrito nas extremidades.

Segundo Vokey et al (2001), a redução do atrito das articulações com o material de cama influencia positivamente sobre a severidade das lesões, possibilitando a cicatrização e a renovação tecidual.

Os resultados encontrados não se assemelham aos resultados encontrados nos estudos de Weary e Taszkun (2000) e Barrientos et al (2013), em que mencionam que as chances de lesão jarrete são menores com o uso de baias com areia como material de recobrimento em comparação com colchões. Quando a vaca deita-se ou levanta-se uma grande quantidade de pressão é aplicada sobre os joelhos; camas com base mais rigidas oferecem pouco amortecimento durante esses movimentos (Heyerhoff et al 2014).

Para os dados de locomoção depreendeu-se que, tanto para os animais que estavam alojados em baias com cama de areia quanto para os que estavam em baias com borracha, não houve diferença entre os escores observados no início e ao final do estudo (Tabela 1).

Tabela 1 Mediana (intervalo interquartil) para os escores de gravidade das lesões jarrete e joelho em vacas utilizando baias com cama de borracha ou areia, no início e no final do período experimental.

\begin{tabular}{ccccc}
\hline & \multicolumn{2}{c}{ Areia } & \multicolumn{2}{c}{ Borracha } \\
\cline { 2 - 5 } & Início & Final & Início & Final \\
\hline Jarrete & $1,00(0-1)^{\mathrm{a}}$ & $1,00(1-2)^{\mathrm{a}}$ & $0,50(0-1)^{\mathrm{a}}$ & $1,50(1-2)^{\mathrm{a}}$ \\
Joelho & $2,00(2-3)^{\mathrm{a}}$ & $2,00(2-3)^{\mathrm{a}}$ & $2,00(1-3)^{\mathrm{a}}$ & $2,00(1-2,25)^{\mathrm{a}}$ \\
Locomoção & $1,50(1-2)^{\mathrm{a}}$ & $1,00(1-2)^{\mathrm{a}}$ & $2,00(1-3)^{\mathrm{a}}$ & $2,00(1-2)^{\mathrm{a}}$
\end{tabular}

Medianas seguidas por mesma letra na linha não diferem entre si $(\mathrm{P}<0,05)$ pelo teste Mann-Whitney.

Os resultados encontrados para locomoção divergem dos observados por Cook et al (2004), Espejo et al (2006) e Norring et al (2008). Os referidos trabalhos sugerem que a utilização da cama de areia reduz a claudicação em relação à vacas sobre esteiras de borracha. O Código Canadense de Prática para o gado leiteiro recomenda "proporcionar piso 
macio, de alta tração em áreas onde o gado ficar por longos períodos" (National Farm Animal Care Council 2009).

A diferença observada em relação à literatura citada, denota que o tempo de exposição aos materiais de cama, no presente estudo, não foi suficiente para alterar a severidade e/ou quantidade tanto para lesões de jarrete, joelho e lesões no casco, e por consequência, manteve os escores de lesões e locomoção nos mesmos patamares.

\section{Conclusões}

Não houve relação entre o tipo de cama e a gravidade de lesões de jarrete, joelho e escore de locomoção. As lesões se mantiveram com os mesmos níveis de escores durante o período estudado, demostrando assim que as duas camas oferecem a mesma condição de conforto em relação a lesões e saúde do casco, pelo menos, em curto prazo de tempo.

\section{Agradecimentos}

Os autores expressam seus agradecimentos à Empresa Brasileira de Pesquisa Agropecuária - EMBRAPA Gado de Leite, e à Coordenação de Aperfeiçoamento de Pessoal de Nível Superior (CAPES).

\section{Referências}

Barberg AE, Endres MI, Salfer JA, Reneau JK (2007) Performance and welfare of dairy cows in an alternative housing system in Minnesota. Journal of Dairy Science 90:1575-1583.

Barker ZE, Leach KA, Whay HR, Bell NJ, Main DCJ (2010) Assessment of lameness prevalence and associated risk factors in dairy herds in England and Wales. Journal of Dairy Science 93:932941.

Barrientos AK, Chapinal N, Weary DM, Galo E, And Von Keyserlingk MAG (2013) Herd-level risk factors for hock injuries in free-stall housed dairy cows in northeastern United States and California. Journal of Dairy Science 96:3758-3765.

Boone R, Bucklin R, Bray D (2009) Comparison of freestall bedding materials and their effect on cow behavior and cow health. American Society of Agricultural Engineers, ASAE 2:739-757.

Carreira XC, Fernández ME, Mariño RA (2009) Indices for estimation of dairy free-stall occupancy. Applied Animal Behaviour Science 119:23-31.

Castro JO, Ferreira RA, Campos AT, Ferreira RA, Yanagi Júnior T, Tadeu HC (2013) Uso de ardósia na construção de celas de maternidade para suínos: II - ambiente térmico e avaliação dos ruídos. Engenharia Agrícola 33:37-45.

Chapinal N, De Passillé AM, Rushen J, Wagner S (2012) Automated methods for detecting lameness and measuring analgesia in dairy cattle. Journal of Dairy Science 93:2007-2013.

Cerqueira JL, Araújo JP, Sorensen JT, Ribeiro JN (2011) Alguns indicadores de avaliação de bem-estar em vacas leiteiras - revisão. Revista Portuguesa de Ciências Veterinárias 110:5-19.
Cook NB, Bennett TB, Nordlund KV (2004) Effect of free stall surface on daily activity patterns in dairy cows with relevance to lameness prevalence. Journal of Dairy Science 87:2912-2922.

Espejo LA, Endres MI, Salfer JA (2006) Prevalence of lameness in high-producing Holstein cows housed in freestall barns in Minnesota. Journal of Dairy Science 89:3052-3058.

Fulwider WK, Grandin T, Garrick DJ, Engle TE, Lamm WD, Dalsted NL, Rollin BE (2007) Influence of free-stall base on tarsal joint lesions and hygiene in dairy cows. Journal of Dairy Science 90:3559-3566.

Heyerhoff JCZ, Leblanc SJ, Devries TJ, Nash CGR, Gibbons J, Orsel K (2014) Prevalence of and factors associated with hock, knee, and neck injuries on dairy cows in freestall housing in Canada. Journal Dairy Science 97:173-184.

Kielland C, Ruud LE, Zanella AJ, Østerås AJ (2009) Prevalence and risk factors for skin lesions on legs of dairy cattle housed in free stalls in Norway. Journal of Dairy Science 92:5487-5496.

Lombard JE, Tucker CB, Von Keyserlingk MAG, Kopral CA, Weary DM (2010) Associations between cow hygiene, hock injuries, and free stall usage on US dairy farms. Journal of Dairy Science 93:4668-4676.

Mitev J, Varlyakov I, Miteva T, Vasilev N, Gergovska J, Uzunova K, Dimova V (2012) Preferences of freestall housed dairy cows to different bedding materials. Journal of Faculty of Veterinary Medicine, 38:135-140.

National Farm Animal Care Council (2009) Code of practice for the care and handling of dairy cattle. Disponível em: <http://www.nfacc.ca/codes-of-practice/dairy-cattle>Acessado em: junho de 2014.

Norring M, Manninen E, De Passillé AM, Rushen J, Munksgaard L, Saloniemi H (2008) Effects of sand and straw bedding on the lying behavior, cleanliness, and hoof and hock injuries of dairy cows. Journal of Dairy Science 91:570-576.

Perissinotto M, Moura D.J, Cruz VF, Souza S.L, Lima KAO, Mendes AS (2009) Conforto térmico de bovinos leiteiros confinados em clima subtropical e mediterrâneo pela análise de parâmetros fisiológicos utilizando a teoria dos conjuntos fuzzy. Ciência Rural 39:1492-1498.

Potterton SL, Green MJ, Harris J, Millar KM, Whay HR, Huxley JN (2011) Risk factors associated with hair loss, ulceration, and swelling at the hock in free stall-housed UK dairy herds. Journal of Dairy Science 94:2952-2963.

Regula G, Danuser J, Spycher B, Wechsler B (2004) Health and welfare of dairy cows in different husbandry systems in Switzerland. Preventive Veterinary Medicine 66:247-264.

Rutherford KMD, Langford FM, Jack MC, Sherwood L, Lawrence AB, Haskell MJ (2008) Hock injury prevalence and associated risk factors on organic and nonorganic dairy farms in the United Kingdom. Journal of Dairy Science 91:2265-2274.

Rushen J, Haley D, De Passillé AM (2007) Effect of softer flooring in tie stalls on resting behavior and leg injuries of lactating cows. Journal of Dairy Science, 90:3647-3651.

Sprecher DJ, Hostetler DE, Kaneene JBA (1997) lameness scoring system that uses posture and gait to predict dairy cattle reproductive performance. Theriogenology 47:1178-1187. 
Vokey FJ, Guard CL, Erb HN, Galton DM (2001) Effects of alley surface on indices of claw and leg health in dairy cattle housed in a free-stall barn. Journal of Dairy Science 84:2686-2699.

Von Keyserlingk MAG, Barrientos A, Ito K, Galo E, Weary DM (2012) Benchmarking cow comfort on North American freestall dairies: Lameness, leg injuries, lying time, facility design, and management for high-producing Holstein dairy cows. Journal of Dairy Science 95:7399-7408.

Weary DM, Huzzey JM, Von Keyserlingk MAG (2009) Board invited review: using behavior to predict and identify ill health in animals. Journal Animal Science 87:770-777.

Weary DM \& Taszkun (2000) Hock lesions and free-stall design. Journal of Dairy Science 83:697-702. 